Exercices Exercices de rhétorique

de rhétorique $\quad 11 \mid 2018$

Sur l'éloge

\title{
Orator christianus (Rome, 1612), « Sur le genre orné »"
}

Traduction de Viviane Griveau-Genest

\section{Carlo Reggio}

Traducteur : Viviane Griveau-Genest

\section{(2) OpenEdition}

1 Journals

Édition électronique

URL : http://journals.openedition.org/rhetorique/717

DOI : $10.4000 /$ rhetorique. 717

ISSN : 2270-6909

Éditeur

UGA Éditions/Université Grenoble Alpes

Édition imprimée

ISBN : 978-2-37747-062-4

Référence électronique

Carlo Reggio, «Orator christianus (Rome, 1612), « Sur le genre orné » », Exercices de rhétorique [En ligne], 11 | 2018, mis en ligne le 17 octobre 2018, consulté le 12 septembre 2020. URL : http:// journals.openedition.org/rhetorique/717 ; DOI : https://doi.org/10.4000/rhetorique. 717

Ce document a été généré automatiquement le 12 septembre 2020.

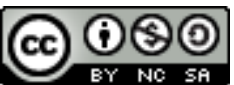

Les contenus de la revue Exercices de rhétorique sont mis à disposition selon les termes de la Licence Creative Commons Attribution - Pas d'Utilisation Commerciale - Partage dans les Mêmes Conditions 4.0 International. 


\section{Orator christianus (Rome, 1612), «Sur le genre orné »}

Traduction de Viviane Griveau-Genest

\section{Carlo Reggio}

Traduction : Viviane Griveau-Genest

\section{Références}

Carlo Reggio, Orator christianus, Rome, Bartholomeus Zanettus, 1612, $2^{\mathrm{e}}$ partie, livre 4, chap. 10 : «In genere exornativo loci » (ou, au titre courant, « De locis generis exornatiui », «Sur les lieux du genre orné »), p. 284-286.

Le texte d'origine ne comporte que deux alinéas, le second commençant à « Les lieux à partir desquels » (Loci vnde aliquod), avec manchette marginale en regard. Pour notre part, nous introduisons un alinéa dès lors qu'une manchette figure en mention marginale.

\section{Sur le genre orné 1}

Commençons ${ }^{2}$ par l'ornement ${ }^{3}$ qui repose entièrement ou principalement sur les louanges. Ce genre semble relever principalement de la voie unitive - nous l'avons expliqué ailleurs - ainsi que de la contemplation. Celle-ci est, en effet, principalement contenue sous la catégorie de l'honnête, et ce à l'inverse de la voie illuminative ; ce sont en effet les exemples des saints et les louanges qui servent de préceptes et d'exhortations pour autrui. Or tous ces éloges doivent viser la persuasion. Parmi eux, le panégyriste [encomiastes] doit se proposer à lui-même : premièrement, l'admiration de la grâce de Dieu, de sa vertu ainsi que de sa divine libéralité envers les saints, à 
commencer par la gloire et la louange du Seigneur Christ ; deuxièmement, la sainteté de celui que l'on célèbre ; troisièmement, l'imitation, en montrant, surtout, que les vertus sont faciles à pratiquer [p. 285] suivant l'exemple des saints, qui étaient des hommes faits exactement de la même pâte ${ }^{4}$ que nous.

Les rhéteurs ${ }^{5}$ ont coutume de présenter plusieurs lieux de la louange, parmi lesquels les parents, les ancêtres, la patrie, la noblesse de la lignée [genus], la richesse, les amis, les honneurs, le pouvoir, l'éducation, la bonne santé du corps, la beauté, la vigueur, les qualités de l'esprit, qu'elles proviennent de la nature, comme l'intelligence, la mémoire, le talent, l'éloquence, ou qu'elles proviennent du travail, comme la science, les vertus, la prudence, la justice, le courage, la tempérance, les actions et les paroles exceptionnelles [facta dictaque] ainsi que le type de mort et tout ce qui peut advenir après la mort ${ }^{6}$. Notre orateur toutefois n'est pas astreint à ces lieux et pour certains, c'est à peine s'il doit les aborder. C'est l'avis de saint Basile : «L'école sacrée, dit-il, ne suit point les règles, ni les préceptes des rhéteurs », et il ajoute un peu plus bas : « La règle en matière de célébration [Encomiorum lex] est de montrer la dignité de la patrie, la famille, l'origine, la première éducation. Notre dogme laisse de côté ces aspects car ils sont superflus? 7 . " Nous avons plutôt coutume de célébrer nos saints pour leur mépris des richesses, des dignités, des honneurs, de la noblesse et d'autres choses semblables. Ainsi, Jérôme méprise-t-il une noblesse [genus] qu'Héliodore avait négligée ${ }^{8}$, et il loue Marcella au motif qu'elle est devenue plus noble en méprisant sa noblesse et que, quoique puissante par son patrimoine, elle s'en est affranchie, s'étant plus encore signalée par sa pauvreté [paupertate insignior ${ }^{\circ}$ ].

On peut ${ }^{10}$ cependant traiter ce lieu de la noblesse selon la manière remarquable dont usent certains Pères. Grégoire de Nazianze enseigne souvent que la noblesse parmi les Chrétiens doit être pesée et estimée individuellement à l'aune des mérites de chacun. On trouve beaucoup d'éléments en ce sens dans l'éloge de sa sœur Gorgonie ${ }^{11}$ et dans celui de Héron ${ }^{12}$. Dans le discours aux Ariens ${ }^{13}$, il établit la véritable noblesse dans le fait de conserver l'image et la ressemblance de Dieu, à l'inverse de ceux qui, ignobles, se rendent semblables au diable. De plus, il énumère clairement quatre genres de noblesse et il distingue la vraie de la fausse ${ }^{14}$. Quant à Cyprien, il loue de très belle manière, dans le Martyre de Celerinus, la noblesse des martyrs qui lui sont apparentés ${ }^{15}$. Saint Ambroise, lui, dans son commentaire sur Luc, enseigne en s'appuyant sur les livres sacrés que chez ceux qui méritent l'éloge il convient de louer non seulement les mœurs, mais encore leurs parents : "ainsi, dit-il, est-ce à la manière d'un héritage transmis qu'une pureté sans tache distinguera ceux que nous voulons célébrer ${ }^{16}$.» Toute noblesse profane doit donc être méprisée plutôt qu'encensée [magnifacienda] par l'orateur chrétien; en revanche, celle qui procède de la vertu des parents peut à bon droit faire l'objet d'une louange. Du reste, dans les louanges, on peut distinguer de nombreux sujets d'éloges [species Encomiorum] : Dieu, les mystères de la nouvelle Loi, les anges, les saints et tout particulièrement la sainte Vierge, les états, comme l'état religieux ou l'état sacerdotal. On loue parfois des vivants : dans ce cas, toutefois, on doit se garder de tout ce qui pourrait être suspecté de vanité ou de flagornerie.

4 Les vertus ${ }^{17}$ offrent une matière remarquable pour les louanges [p. 286] et nous les exaltons [exornamus] particulièrement chez les saints. Il s'agit de: la Prudence, qui comprend la mémoire, l'intelligence et la prévoyance ${ }^{18}$; la Justice, dont relèvent la religion envers Dieu, la piété envers les parents, le respect, la gratitude, la sincérité, la libéralité19 ; la Tempérance, à laquelle se rattachent de nombreuses sortes de vertus, 
tout comme nombre de vertus relèvent du courage - nous les avons exposées ailleurs, nous ne les répétons pas ici. Il nous faut néanmoins louer bien davantage les vertus chrétiennes que sont la Foi, l'Espérance, la Charité, la grâce, les dons de l'Esprit saint. Il nous faut louer les actions et les paroles exceptionnelles, les charges administrées avec sagesse pour la gloire de Dieu et le profit du peuple chrétien. Il nous faut louer les œuvres de Dieu, ses bienfaits, ses miracles. On peut lire de nombreux discours de ce type émanant d'hommes éminemment respectables: Grégoire de Nazianze sur Athanase, sur Basile, sur son père Grégoire, sur son frère Césaire, sur sa sœur Gorgonie, sur Cyprien ${ }^{20}$. Basile : sur sainte Julitte, sur Barlaam, sur Mamas ${ }^{21}$. Chrysostome : sur Job, Élie, les Macchabées, Susanne ${ }^{22}$. Jérôme fait l'éloge [Encomia] d'un grand nombre de personnes dans ses œuvres : il célèbre Paule, Marcella, Bonose, Fabiola, Lucinus ${ }^{23}$. Les Saintes Écritures regorgent aussi de louanges décernées aux justes, un privilège que l'on trouvera dans divers passages ${ }^{24}$, à partir de l'incipit des Psaumes de David, «Heureux l'homme qui ne s'est point laissé aller à suivre le conseil des impies ${ }^{25}$ »: l'Ecclésiastique, "Louons ces hommes pleins de gloire dans leurs descendants ${ }^{26}$ "; le premier livre des Maccabées fait l'éloge de leur grand courage ${ }^{27}$; le dernier chapitre des Proverbes, «Qui trouvera une femme forte ? Elle est plus précieuse que ce qui s'apporte de l'extrémité $d u$ monde ${ }^{28}$. $»$ Le Christ pendant sa vie sur terre a loué nombre de personnes. Son éloge de Jean-Baptiste est soigneusement composé ${ }^{29}$ ( « Jésus commença à parler de Jean au peuple ${ }^{30} "$ ), tout comme celui de Marie-Madeleine la pécheresse, lors du repas chez le Pharisien ${ }^{31}$. On peut trouver des louanges adressées à Dieu dans divers passages des Saintes Écritures, louanges tirées des nombreux et très grands attributs qui lui sont donnés, ainsi que des effets [ab effectis] qui sont ses œuvres ${ }^{32}$. On loue non seulement les personnes mais aussi les actions et les hauts faits, ce qui est le point principal pour louer des personnes ${ }^{33}$. Ainsi Chrysostome exalte-t-il [extollit] l'hospitalité d'Abraham, la patience de $\mathrm{Job}^{34}$.

5 Les lieux ${ }^{35}$ à partir desquels tel ou tel fait peut être amplifié sont : l'honnête, l'utile, l'agréable, le pieux, ainsi que les circonstances ${ }^{36}$. Par exemple, si je veux amplifier [exaggerare] les souffrances du Christ en croix, j'examinerai la personne de celui qui souffre, pour qui cela se fait, quelle est l'intensité du supplice, où et quand cela a lieu. Je ne dois pas seulement exalter [exornamus] les personnes et leurs vertus, mais bien d'autres choses: Tertullien et Cyprien écrivent l'éloge de la patience ${ }^{37}$, Chrysostome, l'éloge de l'aumône, parce qu'elle se trouve être "le plus fructueux de tous les $\operatorname{arts}^{38}$ ". Jérôme ${ }^{39}$, Ambroise et Augustin font des traités à la louange de la virginitéte ${ }^{40}$. Le même Jérôme $^{41}$ ainsi que Basile ${ }^{42}$ célèbrent la vie solitaire.

\section{NOTES}

1. Nous remercions pour cette traduction Cédric Giraud pour sa relecture attentive et stimulante et Anne Régent-Susini ; nous leur sommes grandement redevable. Nous remercions également Christine Noille et Francis Goyet pour leur révision des notes. 
2. C'est-à-dire: «Avec ce chapitre, sur le genre orné, commençons le nouvel ensemble que représentent les chapitres sur les lieux des divers genres (orné, suasoire, etc.) mentionnés à la fin du chapitre précédent. »

3. La traduction par le doublet sémantique "ornement/orné » ne nous apparaît guère satisfaisante pour le français, mais ce choix permet de rendre sensibles les liens conceptuels qu'entretient le présent passage avec la catégorie rhétorique de l'ornatus. Sophie Conte, traduisant de la même manière l'expression chez Caussin, note qu' «il y a presque confusion, dans le le nom même de genus exornativus, entre genre oratoire et genre de style " («La rhétorique sacrée dans les Eloquentiae sacrae et humanae parallela ", dans Nicolas Caussin : rhétorique et spiritualité à l'époque de Louis XIII ; actes du colloque de Troyes (16-17 octobre 2004), Berlin, Lit Verlag, 2007, p. 276).

4. La traduction présente ne permet pas de rendre précisément compte de la présence en creux de la notion de massa peccati (la pâte ou l'argile du péché) issue de L'Épître aux Romains 9, 21 et qu'Augustin a formalisée dans ses textes de controverse contre les Pélagiens.

5. Manchette marginale de l'auteur : «Points principaux [capita] de l'éloge. » - Le mot de capita est un synonyme habituel de loci, « lieux».

6. Souvenir de Quintilien, Institution oratoire, III, 7, 17 (trad. Cousin, Paris, Les Belles Lettres, C.U.F., 1976): «On n'a pas toujours la chance de traiter du temps qui suit la mort du personnage » («Tempus, quod finem hominis insequitur»). Si oui, l'orateur évoque les honneurs reçus post mortem par la personne louée ou sa gloire auprès de la postérité.

7. Note de l'auteur: «Dans son discours faisant l'éloge de Gordius de Césarée.» - Basile de Césarée, Homélie 18, Sur le martyr Gordius, § 2 (1 Bibliotheca Hagiographica Graeca, 703 ; Patrologia graeca, vol. 31, col. 492, B-C) ; pour une traduction française, voir Sermons de S. Basile le Grand, Paris, André Pralard, 1691, "Panégyrique de Saint Gordius martyr, soldat de Césarée ", p. 220-235, à qui nous reprenons la traduction de la première phrase (p. 221); celle de la seconde phrase y est plus développée (graphies modernisées, ici et ensuite) : "Quand on veut faire un éloge, il faut rechercher la patrie de celui qu'on loue, fouiller dans les siècles passés, pour trouver l'antiquité de son origine, examiner soigneusement l'éducation qu'on lui a donnée dès ses plus tendres années; pour louer les Saints nous rejetons tout ce qui est étranger, et nous ne faisons mention que de leurs vertus personnelles."

8. Note de l'auteur : «Dans son éloge funèbre [de Népotien] adressé à Héliodore, Lettre 2.» Jérôme, Lettre 60 sur la mort de Népotien («Ad Heliodorum epitaphium Nepotiani »), § $8:$ : Les rhéteurs prescrivent que l'on remonte très haut jusqu'aux ancêtres de celui qu'on doit louer [...]. [...] Pour moi, ces avantages charnels qu'il a toujours personnellement méprisés, je n'en ferai pas état ", trad. J. Labourt, dans Saint Jérôme, Lettres, Paris, Les Belles Lettres, C. U. F., t. III, 1953.

9. Note de l'auteur : «Dans sa lettre à Marcella, Lettre 16. » - Jérôme, Vie de Marcella (ou Lettre 127, "À la religieuse Principia, sur la vie de sainte Marcella »), §1. Voir la traduction de Jérôme Labourt, dans Saint Jérôme, Lettres, Paris, Les Belles Lettres, C. U. F., t. VII, 1961 : « ce n'est pas selon les méthodes des rhéteurs que je vais la célébrer, en mettant en lumière l'illustration de sa famille, l'honneur d'un sang altier et sa généalogie qui descend parmi les consuls et les préfets du prétoire. Je ne louerai rien en elle qui ne soit bien à elle, mais ce qui est d'autant plus noble que, en méprisant la richesse et la noblesse, elle s'est ennoblie par la pauvreté et l'humilité [paupertate et humilitate nobilior]. " La formulation de Reggio fait également écho à un autre passage de Jérôme, sur la mort de sa disciple sainte Paule (Epitaphium Sanctae Paulae, Lettre 108, I, 1) : « potens quondam diuitiis sed nunc Christi paupertate insignior [...]", "puissante jadis par sa richesse, mais plus insigne à présent par la pauvreté du Christ » (trad. J. Labourt, op. cit., t. V, 1955).

10. Manchette marginale de l'auteur: «La noblesse: sur la manière dont on peut en faire l'éloge. "

11. Voir Grégoire de Nazianze, Discours 6-12, éd. M.-A. Calvet-Sebasti, Paris, Éditions du Cerf, 1995, Discours 8 , « Discours funèbre pour sa sœur Gorgonie ». 
12. Note de l'auteur : "Dans son discours sur Maxime.» - Grégoire de Nazianze, Discours 25, « Sur le philosophe Héron » (Maxime Héron d'Alexandrie), dans Discours 24-26, éd. J. Mossay, Paris, Éditions du Cerf, 1981.

13. Voir Grégoire de Nazianze, ibid., Discours 26, « Deuxième sermon sur lui-même ».

14. Voir ibid., § 10 : «En effet, il y a trois genres de noblesse [en grec, genos]. La première tire son principe d'en haut : par elle, nous sommes tous également nobles, puisque nous avons été faits à l'image de Dieu. La seconde dérive de la chair, et je ne sais si l'on peut être noble par ce qui va de pair avec la corruption. La troisième se reconnaît d'après le vice et la vertu : de celle-ci, nous participons tous plus ou moins, me semble-t-il, dans la mesure où nous entretenons ou corrompons l'image de Dieu. Cette noblesse-là, celui qui est vraiment sage et philosophe l'aimera. Quant à la quatrième noblesse, celle que confèrent les lettres et les décrets, je me donnerai la peine d'en parler lorsque je confondrai les fards avec la beauté et que j'aurai du respect pour un singe dressé à faire le lion. » Traduit dans $\mathrm{P}$. Boulhol, «Les nouvelles règles de l'éloge selon les Pères de l'Église grecs: le refus de l'orgueil familial ou nobiliaire », URL: https:// ch.hypotheses.org/589\#_ftn34

15. Note de l'auteur : «Lettre 34. » - Lettre « 34 » dans l'édition des Opera de saint Cyprien (Paris, S. Nivelle, 1603, p. 67-68), et actuelle lettre 39, III : «Il marche sur les traces des siens, il rejoint dans l'honneur que leur fait la divine bonté, ses parents et ses proches » (trad. du chanoine Bayard, dans Saint Cyprien, Correspondance, Paris, Les Belles Lettres, C. U.F, 1945). Sur le personnage de Celerinus selon Cyprien, voir Y. Duval, "Celerinus et les siens d'après la correspondance de Cyprien (Ep. 21-23, 37, 39) », Revue des Études Augustiniennes, 47, 2001, p. 33-62. Cyprien ne loue pas la nobilitas de Celerinus au sens d'appartenance à une famille connue, mais au sens d'origine glorieuse ("de origine gloriosa»), en raison des martyrs que compte sa famille, à commencer par son aïeule Celerina et ses oncles. Du reste, le mot nobilitas n'apparaît qu'une seule fois dans sa correspondance, contre treize occurrences de claritas. Voir C. Brunet, «L'expression du pouvoir dans la correspondance de Cyprien : étude sémantique des noms en -tas ", Dialogues d'histoire ancienne, 2009/2 (35/2), p. 133-162 ; URL : https://www.cairn.info/revue-dialogues-dhistoire-ancienne-2009-2-page-133.htm. Insistant sur cette unique occurrence de nobilitas, Reggio établit implicitement un lien avec la tradition païenne de l'éloge, tout en la reconfigurant.

16. Note de l'auteur : "Sur Luc, livre I, chap. 1. - Ambroise de Milan, Traité sur l'évangile de saint Luc, trad. G. Tissot, Paris, Cerf, 1956, p. 54. Nous mettons les guillemets en fonction de l'incise « dit-il ». Mais, en réalité, toute la séquence qui commence à "chez ceux qui méritent » est reprise d'Ambroise.

17. Manchette marginale de l'auteur : « Nous louons les vertus. »

18. C'est la tripartition de Cicéron, De l'Invention, II, 160 : «Partes eius: memoria, intellegentia, prouidentia. "

19. Cicéron, De l'Invention, II, 161 (parties du droit naturel ou naturae ius) : « ut religionem, pietatem, gratiam, uindicationem, obseruantiam, ueritatem. » Nous reprenons la traduction de G. Achard pour obseruantia, gratia et ueritas (Paris, Les Belles Lettres, C. U. F., 1994). Reggio supprime de la liste la uindicatio ou désir de vengeance, et il ajoute la libéralité.

20. «Panégyrique de saint Athanase » (discours 21) ; « Oraison funèbre de saint Basile » (discours $43)$; «Oraison funèbre de son père Grégoire l'Ancien» (discours 18); "Discours funèbre en l'honneur de son frère Césaire " (discours 7); "Discours funèbre pour sa sœur Gorgonie » (discours 8, cité ci-dessus, voir la note 11); « En l'honneur de saint Cyprien » (discours 24).

21. Basile de Césarée, "Panégyrique de sainte Julitte martyre», Patrologia graeca, vol.31, col. 237-262, trad. dans Sermons de S. Basile le Grand, op. cit., p. 182-204; «Panégyrique de saint Barlaam martyr », Patrologia graeca, homélie 17, vol. 31, col. 483-490 (trad. dans ibid., p. 215-219) ; «Panégyrique de saint Mamas martyr », Patrologia graeca, homélie 23, vol. 31, col. 589-600 (trad. dans ibid., p. 327-335) - Mamas, ou Mammès ; Reggio écrit Mamante, une des variantes, plus près du texte grec, Mamanta. 
22. Sur Job : quatre homélies, dans Patrologia Graeca, vol. 56, col. 563-582 (classés par la Patrologia parmi les spuria, c'est-à-dire parmi les œuvres faussement attribuées à Chrysostome). Sur Élie : Patrologia Graeca, vol. 51, col. 337-348 et vol. 56, col. 583-586 (celui-ci classé parmi les spuria). Sur les Maccabées : trois homélies, Patrologia Graeca, vol. 50, col. 617-628 (traduction française dans Saint Jean Chrysostome, Euvres complètes, éd. Jeannin, Grenoble, Prudhomme et Giroud, t. III, 1864, p. 367-375). Sur Susanne: Patrologia Graeca, vol.56, col.589-594 (classé parmi les spuria). La traduction latine des œuvres de Jean Chrysostome (Opera, Paris, S. Nivelle, t. I, 1581, dérivant de celle de Venise citée à la note 38) attribue bien au saint les trois homélies sur Job (col. 577-607), les trois sur Élie (col. 559-564) et celle sur Susanne (col. 1413).

23. Jérôme : Paule, Lettre 108 et Marcella : Lettre 127 (citées ci-dessus, voir la note 9). Bonose : Lettre 4, § 4-5. Fabiola : Lettre 77 (éloge funèbre, adressé à son mari). Lucinus : Lettre 75 (éloge funèbre, adressé à son épouse).

24. Littéralement: " louanges décernées aux justes, dont on trouvera les privilèges dans divers passages " (laudibus iustorum, quorum priuilegia passim inuenies).

25. Note de l'auteur: «Psau. 1. »-Psaumes, I, 1 (ici et ensuite, nous reprenons la traduction de Lemaître de Sacy).

26. Note de l'auteur : «Eccl. 44.» - Ecclésiastique, 44, 1 (traduction Sacy modifiée, le texte de la Vulgate étant Laudemus viros gloriosos et parentes nostros in generatione sua).

27. Note de l'auteur: «I. Mach. 8. » - Lire sans doute « 3 » (confusion typographique habituelle). L'incipit de Maccabées, 1, 3 (versets 1-9) fait en effet l'éloge de Judas Maccabée.

28. «Mulierem fortem quis inveniet, procul, et de ultimis finibus pretium ejus. »- Proverbes 31,10 . La Vulgate a mal compris ce passage, qui relève du registre évaluatif. La traduction moderne de la Bible de Jérusalem propose ainsi «Une maîtresse femme, qui la trouvera? / Elle a bien plus de prix que les perles!»

29. «Dedita opera » : la traduction "à dessein" (voir Gaffiot, s. v. dedo, 2, dernier sens) pose problème - arriverait-il au Christ de parler sans dessein? Comprendre plutôt: "avec une attention particulière " ("giving particular attention to this point », selon l'oxford Latin Dictionary, s. v. dedo, 3d).

30. Matthieu, 11, 7 (Reggio reprend la Vulgate : « coepit Iesus dicere ad turbas de Iohanne »). L'éloge de Jean-Baptiste va du verset 7 au verset 15. Même éloge dans Luc, 7, 24-28, avec quasiment la même formule (Vulgate : « coepit dicere de Iohanne ad turbas »).

31. Note de l'auteur: «Luc, 7 et 14.» - Il s'agit surtout du chapitre 7 (versets 44-47), car le chapitre 14 décrit seulement un autre repas de Pharisien.

32. La formulation ab effectis renvoie au lieu des effets : on loue Dieu " par ses effets ", c'est-à-dire par ses œuvres visibles (la Nature, etc.), dont il est la cause invisible. Voir par exemple le début du résumé par Sacy d'Ecclésiastique, 43 : «Grandeur de Dieu marquée dans ses ouvrages. Le ciel, le soleil, la lune [...]. »

33. Note de l'auteur: «Eccl. 43. 44.»- on attendrait plutôt Ecclésiastique, 44 à 49 , ces chapitres faisant l'éloge des «hommes pleins de gloire» (cf. ci-dessus note 26) que sont Énoch, Noé, Abraham, Isaac, etc. Abraham cité à Eccl. 44, 20-23 fait sans doute le lien avec la citation suivante, Jean Chrysostome sur Abraham.

34. Sur Abraham : homélie 41 (sur le chapitre 18 de la Genèse), § 3-6, Patrologia Graeca, vol. 53, col. 378-379 (sur Genèse, 18, 1 : traduction française dans Saint Jean Chrysostome, Euvres complètes, op. cit., t. V, 1864, p. 276-280). Sur Job : voir la note 22 ; la traduction latine des œuvres de Jean Chrysostome (Opera, op. cit., col.572-607) regroupe six homélies sous le titre général de «De patientia Iob ».

35. Manchette marginale de l'auteur : «Lieux pour faire l'éloge d'une vertu dans ses œuvres. » Littéralement: "pour faire l'éloge de tel ou tel ouvrage d'une vertu.» La formulation, opus virtutis, renvoie là encore à l'identification entre effets et œuvres (voir ci-dessus la note $32 ; c f$. opus charitatis, « œuvre de charité »). 
36. L'exemple qui suit, sur le Christ en croix, illustre le seul lieu des circonstances, celles de la personne (la question quis? ou, dans le texte de Reggio, "quis sit qui patitur ») et celles du fait (chez Reggio : « pro quibus, quam acerba, vbi et quando »).

37. Tertullien, De la Patience, éd. J.-Cl. Fredouille, Paris, Éditions du Cerf, 1984 ; Cyprien, La vertu de patience, éd. J. Molager, Paris, Éditions du Cerf, 1982.

38. La formule (chez Reggio, "omnium artium quaestuosissima») se trouve au début de l'exhortation du sermon 52 (53) sur l'évangile de Matthieu (15, 21-22), Patrologia Graeca, vol. 58, col.522, §3. Traduction française de P. A. de Marsilly (dans Homelies ou Sermons de S. Jean Chrysostome, Paris, P. Le Petit, t. II, 1666, p. 531, reprise dans Saint Jean Chrysostome, CEuvres complètes, op. cit., t. VII, 1865, p. 408) : «Reprenons donc encore aujourd'hui ce sujet, et faisons voir que la charité est l'art le plus excellent et le plus divin de tous. Car si le propre d'un art est d'avoir pour objet quelque chose qui soit utile; et s'il n'y a rien de plus utile que la charité que nous exerçons envers les pauvres, n'est-il pas clair que la charité est le plus excellent et le plus avantageux de tous les arts? " Dans Divi Ioannis Chrysostomi [...] operum tomus quintus et ultimus (Venise, Dominicus Nicolinus, 1574, f. 265v, G), toute l'exhortation qui clôt le sermon 52 (53) a été autonomisée en "homélie 33 ", avec pour titre "Quod ars est eleemosyna, et omnium artium quaestuosissima», et pour incipit le passage que nous venons de citer. Les trois mots qui terminent le titre dérivent de la fin de la première phrase de Chrysostome, et ce sont aussi ces trois mots que reprend Reggio.

39. Note de l'auteur: "À Démétrias.» - Jérôme, Lettre 130 (dans Saint Jérôme, Lettres, trad. J. Labourt, Paris, Les Belles Lettres, C. U.F., t. VII, 1961, p. 166-193). La note de Reggio permet d'écarter le Libellus de virginitae servanda de Jérôme, autre nom de sa Lettre 22 (à Eustochium).

40. Ambroise, De Virginitate ; Augustin, De sancta Virginitate.

41. Note de l'auteur : "À Héliodore." - Jérôme, Lettre 14, pour engager le moine Héliodore à le rejoindre dans le désert.

42. Note de l'auteur : «À Grégoire de Nazianze. »- Basile de Césarée, Lettre 14 (quatorzième des Lettres antérieures à l'épiscopat), trad. Y. Courtonne dans Saint Basile, Lettres, Paris, Les Belles Lettres, C. U. F., t. I, 1957, p. 42-45.

\section{AUTEURS}

CARLO REGGIO

S.J. (1540-1612) 